\title{
RAPD markers associated with salt tolerance in soybean genotypes under salt stress
}

\begin{abstract}
In order to investigate the influence of genetic background on salt tolerance in soybean (Glycine max), ten soybean genotypes (Pusa-20, Pusa-40, Pusa-37, Pusa-16, Pusa-24, Pusa22, BRAGG, PK-416, PK-1042, and DS-9712) released in India, were selected and grown hydroponically. The 10-day-old seedlings were subjected to $0,25,50,75,100,125$, and 150 $\mathrm{mM} \mathrm{NaCl}$ for 15 days. Plant growth, leaf osmotic adjustment, and random amplified polymorphic DNA (RAPD) analysis were studied. In comparison to control plants, the plant growth in all genotypes was decreased by salt stress, respectively. Salt stress decreased leaf osmotic potential in all genotypes; however, the maximum reduction was observed in genotype Pusa-24 followed by PK-416 and Pusa-20, while minimum reduction was shown by genotype Pusa-37, followed by BRAGG and PK-1042. Pusa-16, Pusa-22, Pusa-40, and DS9712 were able to tolerate $\mathrm{NaCl}$ treatment up to the level of $75 \mathrm{Mm}$. The difference in osmotic adjustment between all the genotypes was correlated with the concentrations of ion examined such as $\mathrm{Na}+$ and the leaf proline concentration. These results suggest that the genotypic variation for salt tolerance can be partially accounted by plant physiological measures. Twenty RAPD primers revealed high polymorphism and genetic variation among ten soybean genotypes studied. The closer varieties in the cluster behaved similarly in their response to salinity tolerance. Intra-clustering within the two clusters precisely grouped the ten genotypes in sub-cluster as expected from their physiological findings. Our study shows that RAPD technique is a sensitive, precise, and efficient tool for genomic analysis in soybean genotypes.
\end{abstract}

Keyword: Genotypes; Molecular markers; RAPD; Salt stress; Soybean 